\title{
Hydrodynamic constraints on the energy efficiency of droplet electricity generators
}

\author{
Antoine Riaud ${ }^{1}$, Cui Wang' ${ }^{1}$ Jia Zhou', Wanghuai $\mathrm{Xu}^{2}$ and Zuankai Wang ${ }^{2}$
}

\begin{abstract}
Electric energy generation from falling droplets has seen a hundred-fold rise in efficiency over the past few years. However, even these newest devices can only extract a small portion of the droplet energy. In this paper, we theoretically investigate the contributions of hydrodynamic and electric losses in limiting the efficiency of droplet electricity generators (DEG). We restrict our analysis to cases where the droplet contacts the electrode at maximum spread, which was observed to maximize the DEG efficiency. Herein, the electro-mechanical energy conversion occurs during the recoil that immediately follows droplet impact. We then identify three limits on existing droplet electric generators: (i) the impingement velocity is limited in order to maintain the droplet integrity; (ii) much of droplet mechanical energy is squandered in overcoming viscous shear force with the substrate; (iii) insufficient electrical charge of the substrate. Of all these effects, we found that up to $83 \%$ of the total energy available was lost by viscous dissipation during spreading. Minimizing this loss by using cascaded DEG devices to reduce the droplet kinetic energy may increase future devices efficiency beyond $10 \%$.
\end{abstract}

\section{Introduction}

Droplet electricity generators (DEG) are designed to harvest the kinetic energy of rain droplets to power small wireless sensors. Despite a 100-fold increase in efficiency over the past few years ${ }^{1-3}$, even state-of-the-art devices only recover $10 \%$ of the kinetic energy of water ${ }^{4}$, as opposed to the nearly $100 \%$ efficiency achieved by hydroelectric dams.

Unlike dams, which extract energy from the mechanical work of water on the hydro-turbines, DEG, and more broadly triboelectric nanogenerators (TENG) harvest energy from charges accumulated on surfaces which are then used to drive an electric current through an external circuit by electrostatic induction ${ }^{5}$. In the case of DEG, the charges are spontaneously created by water at the surface of polymers ${ }^{6-10}$ by an electron-mediated contact electrification ${ }^{11,12}$. The process can be intensified by applying

\footnotetext{
Correspondence: Antoine Riaud (antoine_riaud@fudan.edu.cn)

${ }^{1}$ State Key Laboratory of ASIC and System, School of Microelectronics, Fudan University, Shanghai 200433, China

${ }^{2}$ Department of Mechanical Engineering, City University of Hong Kong, Hong Kong 999077, China
}

a voltage across the polymer layer ${ }^{13}$. In the latest studies, a grounded metallic electrode is placed underneath the polymer and is connected to a small metallic strip on the top (see Fig. 1). According to the present understanding ${ }^{2,14}$, this sandwiched structure then behaves as a biased capacitor. Upon contact with water, the capacitor is discharged through the load, which releases the electrostatic energy that was stored previously ${ }^{2}$. Meanwhile, mobile charges accumulate at the water-polymer interface. When the droplet recedes, those charges are detached from the interface and forced to return to the bottom electrode ${ }^{4}$. While this model predicts the transfer of charges through the DEG with a remarkable accuracy ${ }^{2}$, it does not consider the hydrodynamic side of the picture. Yet, the harvested electrical energy accounts at best for $10 \%$ of the initial droplet energy, meaning that, in our present understanding, at least $90 \%$ of the droplet energy is unaccounted for.

A comprehensive DEG model would consider (i) the electrical process at play, (ii) the hydrodynamics (iii) the electrochemical charge stability and (iv) the electrohydrodynamic coupling. Since only $10 \%$ of the DEG 


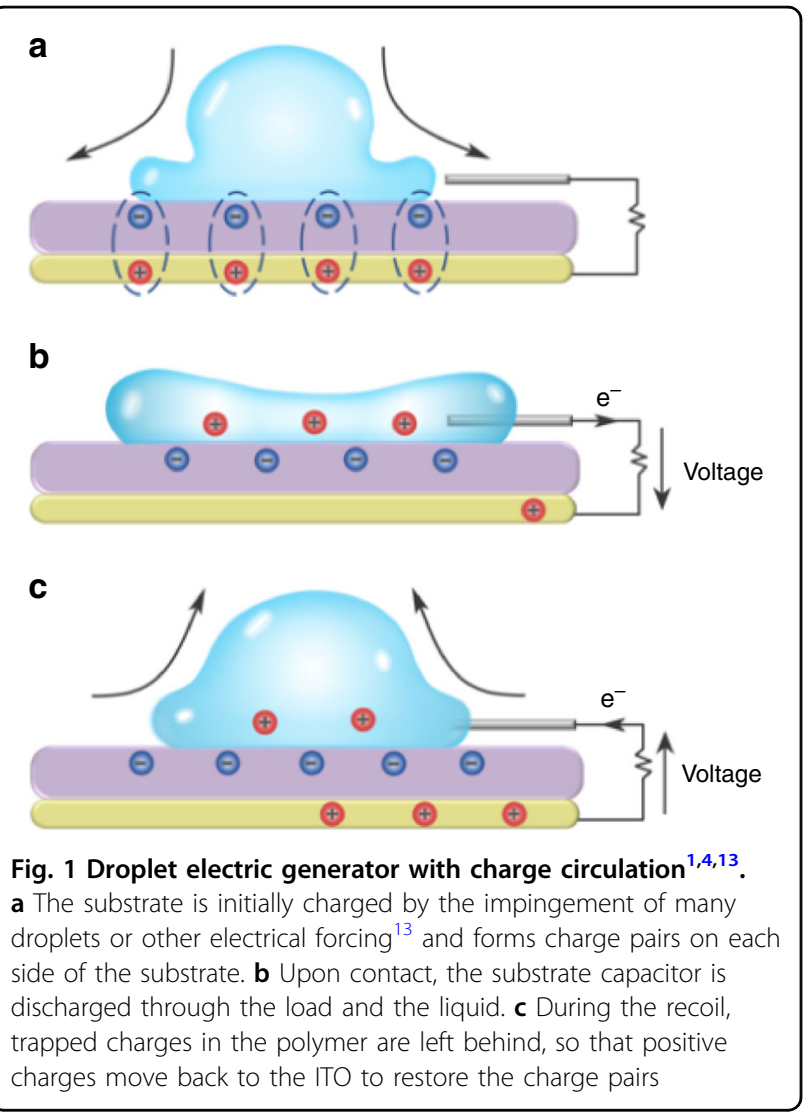

energy is electrical ${ }^{4}$, we neglect the electrohydrodynamic coupling and focus mainly on the hydrodynamic process. Indeed, droplet impacts have been shown to obey the same dynamics regardless of the susbstrate charge ${ }^{1}$. In this simplified view, the DEG hydrodynamics are exactly those of a droplet impacting an inclined plane. Even in this elementary picture, three effects compete to dominate the droplet dynamics: inertia, surface forces, and viscous dissipation. For a spherical droplet of radius $a$, density $\rho$, viscosity $\mu$, surface tension $\gamma$ falling at a velocity $U_{0}$, the ratio of kinetic energy to viscous work is approximately the Reynolds number $\operatorname{Re}=\frac{\rho a U_{0}}{\mu}$, while the ratio of kinetic energy to surface energy is connected to the Weber number $\mathrm{We}=\frac{\rho U_{0}{ }^{2} a}{\gamma}$. During impacts, the liquid spreads into a thin lamella on the solid where most of the energy conversion occurs ${ }^{15}$. It was noticed quite early that a large fraction of the droplet energy is lost during impact ${ }^{16}$. This loss was attributed to viscous dissipation within the lamella ${ }^{16-18}$ until experiments of low-viscosity droplet impacts on super-hydrophobic and slippery substrates suggested that a large fraction of the energy was actually converted to internal kinetic energy (akin to turbulence $)^{19-21}$. Several recent works have since attempted to bridge the gap between these two regimes ${ }^{15,22,23}$. The numerical simulations of Wildeman et al. ${ }^{15}$ are of particular interest for this study, as they show the location of viscous losses within the droplets during impacts, and quantify the fraction of energy dissipated as viscous work and internal kinetic energy over the entire spreading step with no-slip and free-slip boundary conditions. According to this model, nearly half of the initial kinetic energy of low-viscosity droplets is lost as viscous dissipation in the lamella during spreading, regardless of the slip length, impact velocity and fluid viscosity.

The stability of the lamella formed during the impact is also a key concern for DEG. Intuitively, a splashing droplet releases some surface and kinetic energy in the form of ejected daughter droplets. Lamella breakup depends on a competition between a destabilizing suction and lubrication forces that lift it from the substrate, and a restoring capillary force that pulls the liquid back into the bulk of the droplet ${ }^{24}$. When the restoring force is overwhelmed by the other two, the lamella detaches and breaks into smaller droplets, resulting in splashing ${ }^{25}$. Even if the lamella remains stable and recedes, the liquid film itself may have thinned past its own stability limit and rupture into lower-energy liquid islands ${ }^{26}$.

In this paper, we investigate the consequences of the hydrodynamic phenomena on the operating conditions and efficiency of DEG devices. Even though most DEG devices can provide energy during the spreading of the drop $^{1,2,4}$, it was experimentally observed that the DEG energy output peaks when the electrode is placed far enough from the droplet impact such that the electrical contact is established at maximum droplet spread ${ }^{1,2}$. Therefore, we will restrict our analysis to these situations where the droplet spreads to its maximum extent before touching the electrode. In the absence of electrical contact, the liquid provides no electrical energy during the spreading phase, and instead the totality of the energy is obtained during the receding of the liquid film. This is supported by a thought experiment where one would drop a neutral conducting disc on the DEG. The biased capacitor would release the same amount of energy without any work from the disc. However, one would need to overcome the Coulomb force between the mobile charges induced in the disc and the underlying polymer to detach the disc from the DEG, thereby attesting of an electromechanical conversion during recoil. We note that this energy is first stored as electrostatic energy in the polymer until a new disc is placed to release it, thereby completing the energy production cycle. The paper is organized as follows. The first part of the paper discusses the hydrodynamic limits of droplet impacts and illustrates these conditions with simple expressions from the extensive literature on normal droplet impacts ${ }^{15-21,23-29}$. Starting from the total energy available from a falling droplet, we will first estimate the maximum impact velocity allowed for DEG devices depending on the droplet volume, then evaluate how this energy is converted 
into surface energy and turbulent/viscous losses during the spreading phase, followed by the recoil phase. In the second part of the paper, we complement this hydrodynamic picture with the electrical model of $\mathrm{Wu}$ et al. ${ }^{2}$ to quantify the energy efficiency of DEG devices. We use experimental data from previous studies ${ }^{1,2,4}$ to explore various operating conditions in a realistic setting (oblique impacts) and point out to possible improvements for this technology.

\section{Results and discussion \\ Fluid mechanics model \\ Available energy}

A falling droplet combines a kinetic energy $\mathcal{K}_{0}=$ $\frac{2 \pi}{3} a^{3} \rho U_{0}^{2}$ and surface energy $\mathcal{V}_{0}=4 \pi a^{2} \gamma$. Neglecting gravity, the minimum energy of a liquid in contact with a solid surface is obtained when the spherical droplet intersects with the solid surface at the Young contact angle $\cos \theta=\frac{\gamma_{s v}-\gamma_{s l}}{\gamma}$. According to volume conservation, this yields ${ }^{30}$ :

$$
\begin{aligned}
& \mathcal{V}_{\text {eq }}=\gamma A_{\text {eq }, \text { cap }}-\gamma \cos \theta A_{\text {eq, base }} \\
& \text { with }: \quad \mathrm{A}_{\text {eq,cap }}=2 \pi \mathrm{R}_{\text {eq }}{ }^{2}(1-\cos \theta) \\
& A_{\text {eq,base }}=\pi R_{\text {eq }}{ }^{2} \sin ^{2} \theta \\
& \text { and } \quad \mathrm{R}_{\text {eq }}=\frac{2^{2 / 3} \mathrm{a}}{\left(2-3 \cos \theta+\cos ^{3} \theta\right)^{1 / 3}}
\end{aligned}
$$

where $A_{\text {eq,cap }}$ and $A_{\text {eq,base }}$ are the cap and base surface area of the droplet at equilibrium, and $R_{\mathrm{eq}}$ is its radius of curvature. This ideal situation is never achieved in practical DEG devices. Therefore, the maximum energy available is obtained by subtracting this lowest possible energy from the initial energy:

$$
\mathcal{E}_{\max }=\mathcal{K}_{0}+\mathcal{V}_{0}-\mathcal{V}_{\text {eq }}
$$

\section{Maximum impact velocity}

The impact velocity is limited by two factors. On the one hand, the droplet may splash, but even without splashing, the film formed by the impacted droplet may still become unstable and rupture. We first recall the splashing conditions according to Riboux and Gordillo ${ }^{25}$. While their study focuses on normal impacts, they mainly discuss the local dynamics of the lamella formed during the droplet impact, which suggests that, at least qualitatively, the splashing mechanism evidenced by these authors may also be relevant for oblique impacts.

When a droplet impacts a solid surface, it forms a lamella that may recoil or break into droplets if splashing occurs. To splash, the lamella must satisfy two conditions: (i) the liquid sheet must be ejected from the solid and (ii) the growing rim at the edge of the lamella must not reconnect with the solid afterwards. Interpreting condition (i) as a force balance yields the ejection ejection time $T_{e}=t_{e} \frac{a}{U_{0}}{ }^{25}$ from the real positive root $\sqrt{t_{e}}$ of the fourthorder polynomial:

$$
\frac{\sqrt{3}}{2 \operatorname{Re}}+\frac{\sqrt{t_{e}}}{W e}=1.1 t_{e}^{2}
$$

Upon ejection, the lamella lifts off with a vertical velocity $V_{v}$. However, the ejected film also recoils with a speed $V_{r}$ due to surface tension, which forms a fast-growing rim that may eventually reconnect with the solid, thereby preventing splashing. A mass balance shows that the rim grows at a rate $b_{\max } V_{r}$, which yields the second splashing condition:

$$
V_{v} \geq b_{\max } V_{r}
$$

where the constant $b_{\max }=0.14$ was determined experimentally by Riboux and Gordillo ${ }^{25}$. Lamella recoil speed $V_{r}$ and vertical speed $V_{v}$ at the ejection time are given by:

$$
\begin{aligned}
& V_{r}=\sqrt{2 \gamma / \rho H_{t}} \\
& V_{v}=\sqrt{\frac{\ell}{\rho H_{t}}} \\
& \text { with } \quad \ell=K_{l} \mu_{g} V_{t}+K_{u} \rho_{g} V_{t}^{2} H_{t} \\
& \text { and } H_{t}=(a \sqrt{12} / \pi) t_{e}^{3 / 2}
\end{aligned}
$$

Here, $H_{t}$ is the lamella film height at $T_{e}$, and $K_{l} \simeq-\left[6 / \tan ^{2} \alpha\right]\left(\log \left(19.2 \lambda_{g} / H_{t}\right)-\log \left(1+19.2 \lambda_{g} / H_{t}\right)\right)$ and $K_{u}=0.3$ are two hydrodynamic coefficients for the suction and lubrication forces that make up the lift force $\ell$. $\rho_{g}, \mu_{g}$ and $\lambda_{g}$ are the gas phase density, dynamic viscosity and mean-free path.

Even in the absence of splashing, the liquid film formed after impact may still rupture. Surprisingly, even though instability of static liquid films is a well-studied topic $^{31-33}$, film rupture immediately after droplet impact has received much less attention than the more spectacular splashing. Diman and Chandra ${ }^{26}$ have studied the disintegration of liquid films formed after high-speed normal collision between a droplet and a wall. While their analysis is rather involved, and depends on the liquid-solid contact angle and the size of defects that trigger the liquid film instability, experimental evidence over a range of contact angles, surface roughness and liquids suggest that the liquid film will rupture if the droplet impacts a solid wall above some critical 


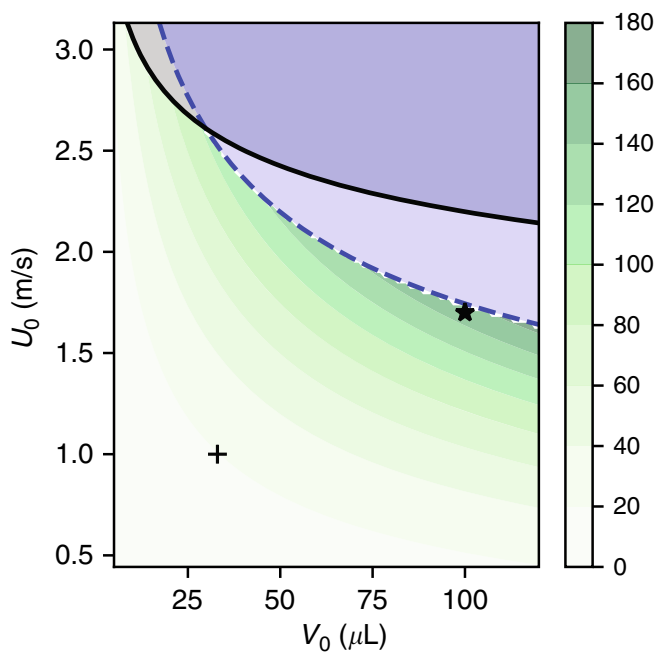

Fig. 2 The available energy and the limiting velocities for splashing and film rupturing. Maximum energy $\mathcal{E}_{\max }$ (in $\mu \mathrm{J}$ ) available from droplets impacting perpendicular surfaces as given by Eq. (5). The splashing (solid line) and film-rupturing (dashed line) limits are given by Eqs. (7) and (12). The markers + and $\star$ represent the experiments of Wu et al. ${ }^{2}$ (energy available $19 \mu \mathrm{J}$ ) and Xu et al. ${ }^{1}$ (energy available $151 \mu \mathrm{J}$ ), respectively

Reynolds number $\operatorname{Re}_{c} \simeq 5000$ :

$$
\operatorname{Re} \geq \operatorname{Re}_{c}
$$

The available energy and the limiting velocities for splashing (Eq. (7)) and film rupturing (Eq. (12)) are shown in Fig. 2. For the sake of simplicity, this figure is computed for deionized (DI) water but would be almost identical for tap water, sea water, rain water and $100 \mathrm{mM}$ $\mathrm{NaCl}$ water solutions as they share very similar mechanical properties, as discussed in Supplementary Information. Note that smaller droplets tend to splash first while larger ones may not splash but their liquid film will rupture nonetheless. The largest theoretical amount of energy while maintaining droplet integrity is obtained for the largest droplets at velocities close to $1.7 \mathrm{~m} / \mathrm{s}$, remarkably close to $\mathrm{Xu}$ et al. ${ }^{1}$.

\section{Efficiency during spreading and receding}

Depending on the impact speed, a sizable fraction of the available energy (Eq. (5)) may be dissipated into turbulent kinetic energy and viscous work during the droplet spreading. This yields the conversion efficiency $\eta_{s}$ :

$$
\eta_{s}=\frac{\mathcal{V}_{\max }}{\mathcal{E}_{\max }}
$$

with $\mathcal{V}_{\max }$ the resulting surface energy at maximum spread $\mathcal{V}_{\max }=\gamma(1-\cos \theta) A_{\max }$, where the surface energy

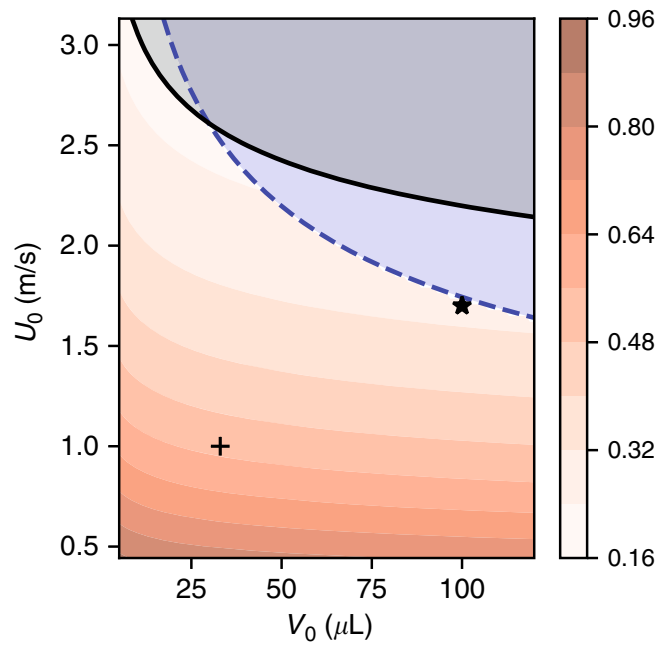

Fig. 3 Mechanical to kinetic conversion efficiency $\eta_{s}(\%)$ according to Eq. (14) for droplets impacting perpendicular surfaces. The splashing (solid line) and film-rupturing (dashed line) limits are given by Eqs. (7) and (12). The markers + and $\star$ represent the experiments of Wu et al. ${ }^{2}$ (spreading efficiency $17 \%$ ) and Xu et al. ${ }^{1}$ (spreading efficiency $30 \%$ ), respectively

$A_{\max }$ may be determined from experimental or simulated droplet impacts, or using simplified models (see refs. ${ }^{18,23}$ for critical reviews on normal impacts, and ref. ${ }^{22}$ for oblique impacts). Among these models, Pasandideh-Fard et al. ${ }^{17}$ have provided a simple and accurate estimate of the maximum spreading diameter of droplets impinging on a perpendicular surface:

$$
a_{\max }=a \sqrt{\frac{\mathrm{We}+6}{\frac{3}{2}(1-\cos \theta)+4 \mathrm{We} \sqrt{\frac{2}{\mathrm{Re}}}}}
$$

In spite of being derived for highly viscous fluids only, this formula was empirically found to work well for lowviscosity fluids as well ${ }^{18,23}$. We refer the reader to Wildeman et al. ${ }^{15}$ for a more physically-sound model at low viscosity. The resulting energy efficiency obtained by combining Eqs. (13) and (14) is shown in Fig. 3. Similarly to Fig. 2, this figure is computed for DI water but would look essentially the same for other diluted water solutions.

During the recoil phase, the surface energy $\mathcal{V}_{\max }$ is split into 3 terms: the viscous work during recoil $\mathcal{W}_{r}$, the electrical work during recoil $\mathcal{W}_{e}$ and the final mechanical energy remaining in the droplet $\mathcal{E}_{\infty}$ :

$$
\mathcal{V}_{\max }=\mathcal{W}_{r}+\mathcal{W}_{e}+\mathcal{E}_{\infty}
$$

Experiments with water drops bouncing on superhydrophobic substrates ${ }^{21,29}$ suggest that the viscous 
dissipation is small during the recoil step, even at high impact velocity, and that most of the surface energy is either restored as external kinetic energy or vibration energy. This is confirmed by the following rough estimate of the droplet viscous dissipation. We first assume that the recoil speed scales as $U_{r}=a_{\max } / \tau_{r}$, with $\tau_{r}$ the contact time between the droplet and the substrate. For superhydrophobic substrates, the contact time, that is a negligible spreading time plus the receding time, is insensitive to the impact velocity ${ }^{19,27}$. Hence, the recoil time scales proportionally to $\tau_{r}=\pi \tau_{h} / \sqrt{2}$ with $\tau_{h}=\sqrt{\frac{\rho a^{3}}{\gamma}}$ the oscillation period of a levitated $\operatorname{drop}^{27,34}$. Using $U_{r}$ as the characteristic recoil velocity, we then compute the volumetric shear rate similarly to Pasandideh-Fard ${ }^{17}$ and obtain a coarse estimate of the viscous work during recoil:

$$
\begin{aligned}
& \mathcal{W}_{r} \simeq \tau_{r} \mu\left(\frac{U_{r}}{\delta}\right)^{2} V_{0} \\
& \text { with : } \delta=\min \left(\mathrm{h}_{\mathrm{bl}}, \mathrm{h}_{\text {film }}\right) \\
& h_{\mathrm{bl}}=\sqrt{\frac{2 \mu \tau_{r}}{\rho}} \\
& h_{\text {film }}=\frac{V_{0}}{A_{\max }}
\end{aligned}
$$

where $h_{\mathrm{bl}}$ and $h_{\text {film }}$ are the hydrodynamic boundary layer and film thickness during recoil.

\section{Electrical model}

\section{Biased capacitor model}

By analogy with a biased capacitor, Wu et al. ${ }^{2}$ have derived the following equation for the charge $q$ driven through the load by the droplet motion:

$$
\frac{d q}{d t}=\frac{1}{R c_{p}}\left(\sigma-\frac{q}{A}\right)
$$

with $\sigma$ the surface charge of the polymer, $c_{p}$ the areal capacitance of the polymer and $R=R_{L}+R_{D}$ the total resistance of the circuit, including the droplet resistance $R_{D}$ and the load $R_{L}$. In the biased capacitor model, $A(t)$ stands for the evolving area of the droplet, but the overlap area of charged polymer in contact with the droplet should be used instead when the polymer charge is nonuniform ${ }^{4}$. We note that this model considers the polymer charge at the time of the droplet impact, regardless of how this charge was generated (such as successive droplet impacts $^{1,35}$ or external charging ${ }^{4,13}$ ).

The energy output reads $\mathcal{W}_{L}=\int_{0}^{t_{c}} R_{L}\left(\frac{d q}{d t}\right)^{2} d t$, where the origin of time $t=0$ is chosen at droplet contact, and $t_{c}$ is the time when the droplet detaches from the electrode.
Using integration by part and substituting Eq. (20) in this integral yields:

$$
\begin{aligned}
& \mathcal{W}_{L}=\eta_{L}\left(\mathcal{E}_{\text {stat }}-\mathcal{E}_{\text {dyn }}\right) \\
& \text { with }: \eta_{\mathrm{L}}=\frac{\mathrm{R}_{\mathrm{L}}}{\mathrm{R}} \\
& \mathcal{E}_{\text {stat }}=\frac{\sigma q\left(t_{c}\right)}{c_{p}} \\
& \mathcal{E}_{\text {dyn }}=\frac{1}{2 c_{p}} \int_{0}^{t_{c}} \frac{d q^{2}}{d t} d t
\end{aligned}
$$

Note that the right-hand side of Eq. (24) cannot be simplified immediately because there is no one-to-one correspondence between $A(t)$ and $q^{2}(t)$. Eq. (21) is made of three parts: an efficiency factor $\eta_{L}$ that accounts for the resistive losses in the liquid, an electrostatic energy $\mathcal{E}_{\text {stat }}$ and a contribution that depends on the dynamics of the charges and droplet geometry $\mathcal{E}_{\text {dyn }}$. Analytical integration of Eq. (20) (see Supplementary Information) shows that $\mathcal{E}_{\text {dyn }}$ is positive only if $q^{2}$ decreases, meaning that this term acts as a generator only when charges are moving out of the droplet.

\section{Available energy}

The droplet exchanges electrical energy with the load twice. First during the discharge of the bottom electrode in the liquid, and then during the recoil step. As stated in the introduction, we restrict our analysis to cases where the droplet spreads to its maximum diameter before contacting the electrode, so that the discharge process goes without energy exchange between the droplet and the DEG: the liquid merely acts as a conductor to release the stored electrostatic energy. Although the discharge step requires no work from the droplet, the droplet-polymer interface becomes increasingly charged thereafter. During the recoil step, the liquid interface area shrinks such that the liquidpolymer interfacial capacitance decreases, which prompts charges to flow back to the bottom electrode. During charge separation, the dry polymer recovers its static charge, which builds up electrostatic energy that will be released by the next droplet. In order to reduce the contact area between oppositely charged surfaces, some electrowetting work must be provided to overcome the electrostatic energy ${ }^{36,37}$. When charges flow back, they provide resistive electrical work through the load but also through the liquid, thereafter called resistive losses:

$$
\mathcal{W}_{\mathrm{R}}=\left(1-\eta_{L}\right)\left(\mathcal{E}_{\text {stat }}-\mathcal{E}_{\text {dyn }}\right)
$$

Upon sufficient recoil, the droplet eventually detaches from the top electrode and the current stops flowing 
through the load. Integrating Eq. (20) shows that the static loss cannot be entirely eliminated unless the droplet area vanishes at $t_{c}$ (see materials and methods). This can be achieved by using non-uniform surface charges ${ }^{4}$. While there is no constraint on the remaining amount of charges (missing charges can be provided from the electrical ground), a highly charged droplet will need to expend more energy to leave the substrate than a neutral one. Therefore, the less charge remains in the droplet, the more energy is available for the load. We will refer to the additional electrostatic expense as the static loss $\mathcal{W}_{\text {stat }}=$ $\frac{q^{2}\left(t_{\infty}\right)}{2 c_{p} A\left(t_{\infty}\right)}$, where $t_{\infty}$ indicates the time the droplet breaks away from the DEG substrate. By conservation of charge, $q\left(t_{c}\right)=q\left(t_{\infty}\right)$, and by conservation of energy the work provided to change the droplet surface area must compensate exactly the variation of $\mathcal{W}_{\text {stat }}$, therefore:

$$
\mathcal{W}_{\text {stat }}=\frac{q^{2}\left(t_{c}\right)}{2 c_{p} A\left(t_{c}\right)}
$$

\section{Main equations}

In summary, the energy extracted from the DEG $\left(\mathcal{W}_{L}\right)$ is the maximum available energy $\mathcal{E}_{\max }$ minus the mechanical $\left(\mathcal{W}_{\text {mech }}\right)$ and electrical $\mathcal{W}_{\text {electr }}$ losses.

$$
\mathcal{W}_{L}=\mathcal{E}_{\text {max }}-\mathcal{W}_{\text {mech }}-\mathcal{W}_{\text {electr }}
$$

The mechanical energy is dissipated during spreading, recoil, and droplet detachment:

$$
\begin{aligned}
& \text { spreading work : } 1-\eta_{s}=1-\frac{\mathcal{V}_{\max }}{\mathcal{E}_{\max }} \\
& \text { recoil work: } \mathcal{W}_{r} \simeq \tau_{r} \mu\left(\frac{U_{r}}{\delta}\right)^{2} V_{0} \\
& \text { untapped mechanical energy : } \mathcal{E}_{\infty}
\end{aligned}
$$

Here, $\mathcal{V}_{\max }$ is obtained experimentally or empirically from the maximum droplet spread $A_{\max }$ and $\mathcal{W}_{r}$ is computed from Eq. (16). The untapped mechanical energy $\mathcal{E}_{\infty}$, attributed to the mechanical energy remaining in the escaping droplet, is obtained from Eq. (15) after computing the electrical work $\mathcal{W}_{e}=W_{\text {electr }}+\mathcal{W}_{L}$. The electric losses are the resistive losses in the liquid and the electromechanical work needed to separate a charged droplet from a charged surface:

$$
\begin{array}{ccc}
\text { resistive losses : } & \mathcal{W}_{\mathrm{R}}=\int_{0}^{t_{c}} R_{D}\left(\frac{d q}{d t}\right)^{2} d t \\
\text { static charge losses : } & \mathcal{W}_{\text {stat }} \quad=\frac{q^{2}\left(t_{c}\right)}{2 c_{p} A\left(t_{c}\right)}
\end{array}
$$

The extracted energy reads:

$$
\mathcal{W}_{L}=\int_{0}^{t_{c}} R_{L}\left(\frac{d q}{d t}\right)^{2} d t
$$

where the charge $q(t)$ traveling through the load is obtained experimentally or by numerically solving Eq. (20). A table of symbols is available in the Supplementary Information.

\section{Discussion}

Figure 2 suggests that large droplets yield the maximum energy within hydrodynamic stability limits. In order to get a more faithful picture under these optimized conditions, we have simulated in OpenFOAM the impact of $100 \mu \mathrm{l}$ droplets on Teflon at various impact velocities (Fig. 4) with an impact angle of $45^{\circ}$ (similar to the one used by Xu et al. ${ }^{1}$ ). We note that this angle has a limited influence on the performance of DEG devices ${ }^{2}$. In agreement with the stability bounds, we observe the onset of film rupture (and not splashing) when the impact velocity exceeds $1 \mathrm{~m} / \mathrm{s}(\mathrm{Re} \simeq 2000)$.

Based on DEG impact videos ${ }^{1,2}$ and our simulations, we evaluate the surface energy $\mathcal{V}_{\max }=\gamma(1-\cos \theta) A_{\max }$ available as the impinged droplet spreads to its maximum diameter. The viscous work during recoil is then deduced from Eq.(16). Similarly to previous studies ${ }^{1,2}$, we optimize the value of $R_{L}$ to maximize the energy output at given $A(t)$ (obtained from experimental videos or simulations). With these optimized parameters, we compute the energy generated per droplet, together with the static and resistive losses (Eqs. (21), (26) and (25)), which yields the total electrical energy available $\mathcal{W}_{e}$. The simulation parameters are given in Table 1 together with the final conversion efficiency, and the relative shares of each energy contribution are shown in Fig. 5.

As shown in Table 1, the experimental efficiency may deviate from the numerical efficiency by up to $50 \%$. Since we used experimental surface areas to predict the DEG efficiency, the deviation must originate from the electrical model (Eq. (20)). Even though this equivalent circuit model $^{2}$ is remarkably accurate for low currents and stands on solid physical grounds, it was not validated for higher currents nor used to predict efficiencies in previous works. Like many physical models, it may fail to resolve singularities such as the current spike upon contact, or possibly nonlinear electro-hydrodynamic couplings or electrical double layer effects during fast discharges. We also note that these very fast current dynamics are difficult to capture experimentally which suggests that an accurate comparison would be very challenging. Nonetheless, the general trend between different DEG architectures is well conserved, which suggests that these calculations may be a useful guide for DEG design.

According to Fig. 5a, the conversion efficiency of impinging droplets depends on the droplet size and impact speed. The impact speed is set as initial condition in the simulations and controlled by the falling height in the experiments ${ }^{18}$. For large droplets impacting at high speed 


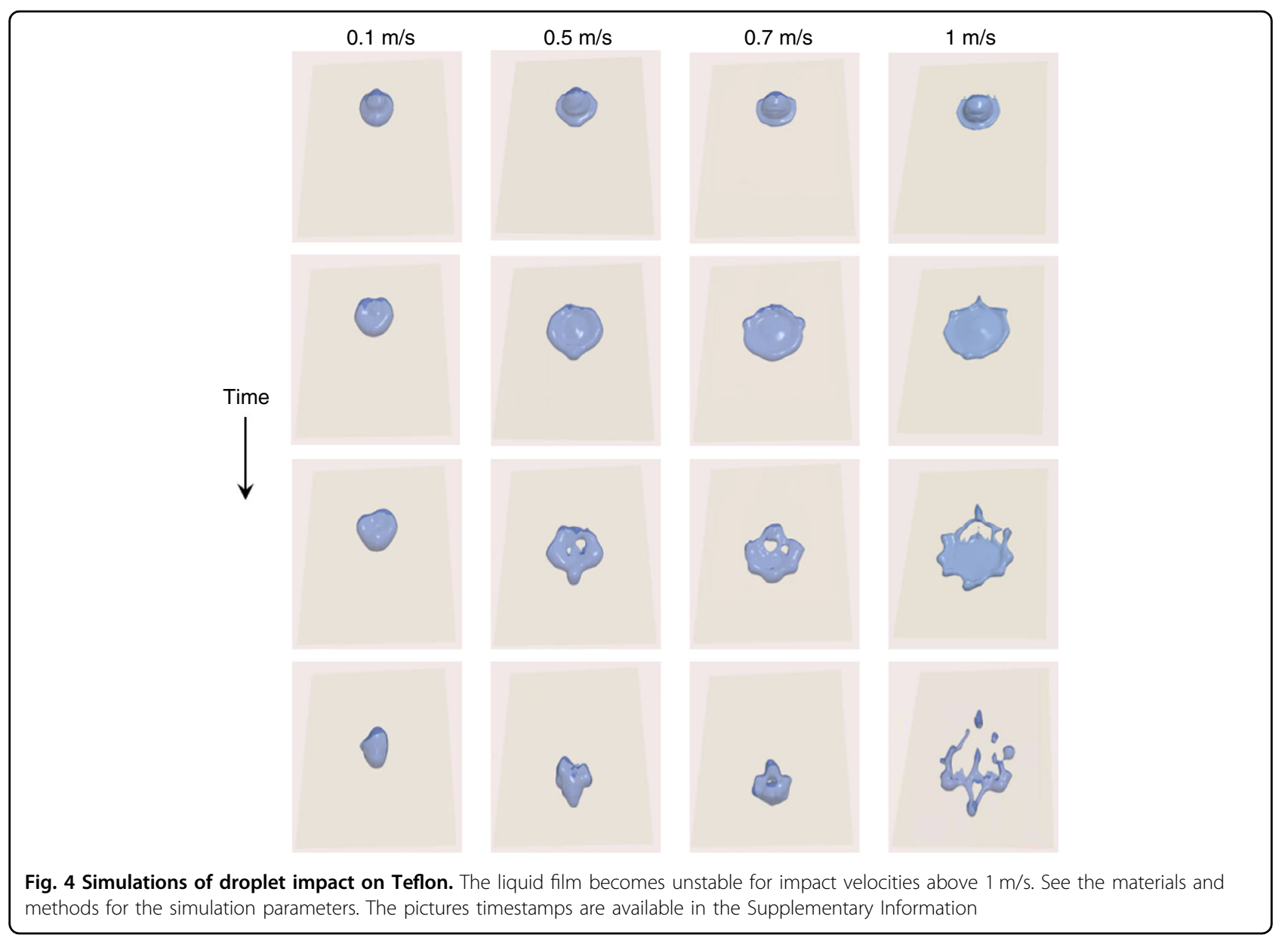

(X5), most of the energy (83\%) is lost as viscous work, in good agreement with Fig. 3 and previous studies ${ }^{15}$. The recoil consumes less than $2 \%$ of the total energy $\mathcal{E}_{\max }$ by itself. The second largest loss contribution $(\simeq 10 \%$ of the total, $74 \%$ of the energy available after recoil) is the difference between the energy after recoil and the electrical energy. This large mismatch is shared across all sizes of droplets regardless of the impact speed, and represents the mechanical energy $\mathcal{E}_{\infty}$ remaining after the droplet detaches from the electrode. By analogy with bouncing droplets ${ }^{21,29}$, it is likely that this energy is a combination of internal kinetic energy and surface vibration energy. Next, an optimized load allows extracting almost $75 \%$ the electrical energy (resistive losses are negligible and the static losses represent $\sim 1 \%$ of $\mathcal{E}_{\max }$ ).

Having identified that most of the energy is dissipated by viscous work and inefficient energy conversion during recoil, we now point to some ways to reduce these losses. The ratio of viscous to capillary work scales as $\mathrm{Ca}=\frac{\mu U_{0}}{\gamma}$ and looks insensitive to the main dimensions of the surface, which suggests that micropatterns which were successful at reducing the droplet spreading and contact time $^{38}$ may not help minimizing the viscous dissipation.
However, our simulations suggest that decreasing the impact velocity from $1.7 \mathrm{~m} / \mathrm{s}(\mathrm{X} 5)$ to $U_{0}=0.1 \mathrm{~m} / \mathrm{s}(\mathrm{X} 1)$ can cut the viscous dissipation during spreading to only $15 \%$ of $\mathcal{E}_{\max }$. This is in line with the improvement of restoring coefficient of bouncing Leidenfrost-levitated droplets at lower speed ${ }^{21}$. Therefore, while high-speed impacts generate more energy, low-speed impacts convert kinetic to surface energy with a much higher efficiency. Nonetheless, low-speed impacts then fail to extract the remaining mechanical energy from the droplet, so that only $5.1 \%$ of $\mathcal{V}_{\max }$ is converted to electricity when $U_{0}=$ $0.1 \mathrm{~m} / \mathrm{s}$ (X1) whereas up to $24 \%$ of $\mathcal{V}_{\max }$ becomes electricity when $U_{0}=1.7 \mathrm{~m} / \mathrm{s}(\mathrm{X} 5)$.

A tentative interpretation is based on the following rough estimate of the energy conversion ${ }^{2}$ :

$$
\mathcal{W}_{L} \simeq \frac{(\Delta A)^{2} \sigma^{2}}{A_{\max } c_{p}}
$$

with $\Delta A$ the difference of liquid-solid surface area between the time when the droplet connects to the electrode and when it breaks away from it. Note that this estimate differs from the $E_{0}=A_{\max } \sigma^{2} /\left(2 c_{p}\right)$ proposed by 

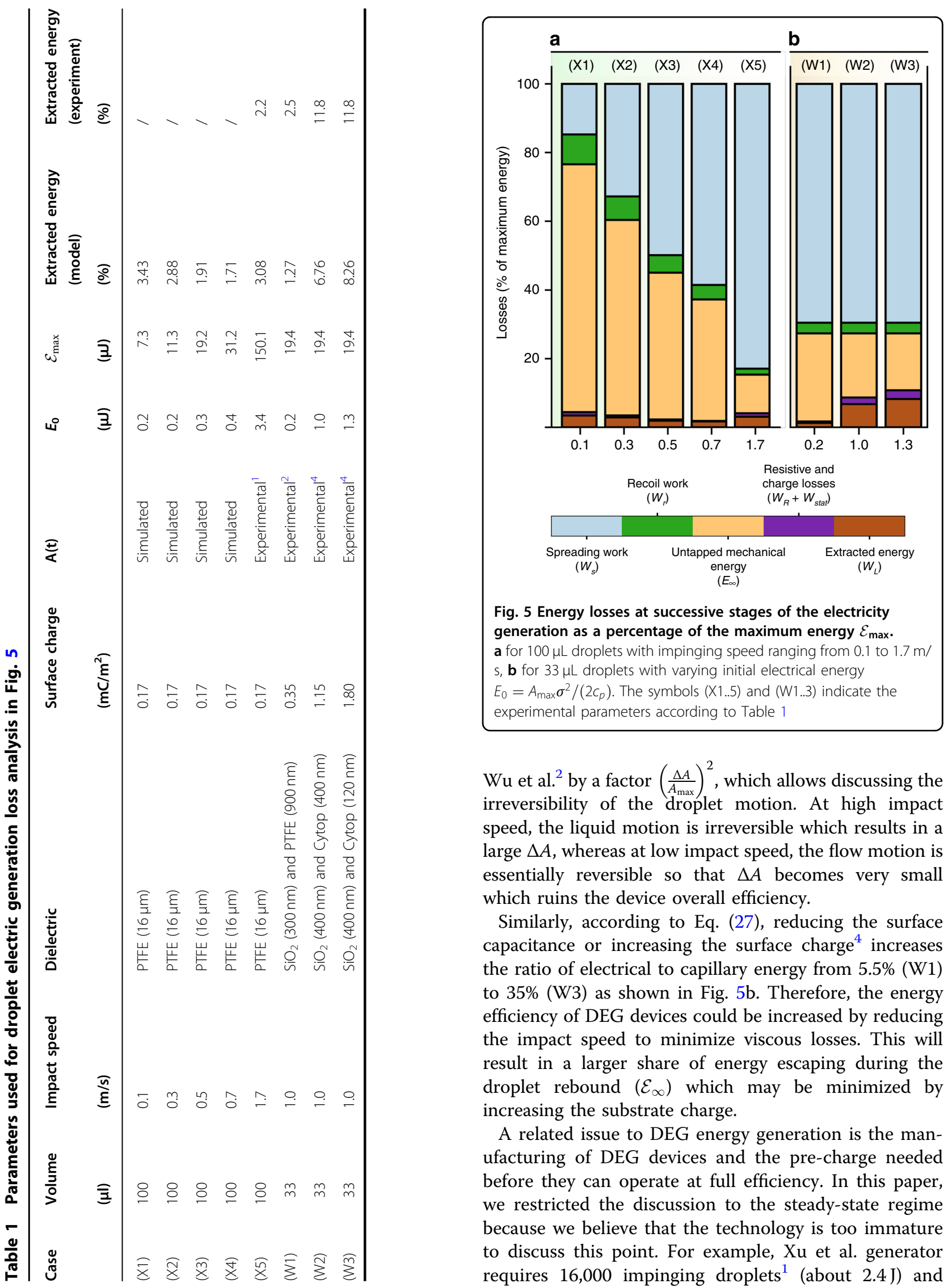

Wu et al. ${ }^{2}$ by a factor $\left(\frac{\Delta A}{A_{\max }}\right)^{2}$, which allows discussing the irreversibility of the droplet motion. At high impact speed, the liquid motion is irreversible which results in a large $\Delta A$, whereas at low impact speed, the flow motion is essentially reversible so that $\Delta A$ becomes very small which ruins the device overall efficiency.

Similarly, according to Eq. (27), reducing the surface capacitance or increasing the surface charge ${ }^{4}$ increases the ratio of electrical to capillary energy from $5.5 \%$ (W1) to $35 \%$ (W3) as shown in Fig. 5b. Therefore, the energy efficiency of DEG devices could be increased by reducing the impact speed to minimize viscous losses. This will result in a larger share of energy escaping during the droplet rebound $\left(\mathcal{E}_{\infty}\right)$ which may be minimized by increasing the substrate charge.

A related issue to DEG energy generation is the manufacturing of DEG devices and the pre-charge needed before they can operate at full efficiency. In this paper, we restricted the discussion to the steady-state regime because we believe that the technology is too immature to discuss this point. For example, Xu et al. generator requires 16,000 impinging droplets ${ }^{1}$ (about 2.4 J) and 
Table 2 Simulation parameters

\begin{tabular}{ll}
\hline Parameters & Value \\
\hline Air kinematic viscosity & $1.48 \times 10^{-5} \mathrm{~m}^{2} / \mathrm{s}$ \\
Air density & $1.0 \mathrm{~kg} / \mathrm{m}^{3}$ \\
Water kinematic viscosity & $1.0 \times 10^{-6} \mathrm{~m}^{2} / \mathrm{s}$ \\
Water density & $1.0 \times 10^{3} \mathrm{~kg} / \mathrm{m}^{3}$ \\
Surface tension & $7.0 \times 10^{-2} \mathrm{~N} / \mathrm{m}$ \\
Contact angle & $114^{\circ}$ \\
\hline
\end{tabular}

$\mathrm{Wu}$ et al. charged their substrates for $15 \mathrm{~min}$ using homogeneous electrowetting-assisted charge injection $(\mathrm{h}-\mathrm{EWCI})^{2,4,13}$ which requires $1.5 \mathrm{~mJ}$ of electrostatic energy in theory, but nearly $81 \mathrm{~kJ}$ in practice due to the $90 \mathrm{~W}$ consumption of the voltage amplifier used in their study. We are hopeful that suitable material choice and optimized industrial setup will dramatically reduce these pre-charge energies.

In this paper, we have used a combination of analytical equations, numerical model and experimental data to map out the energy losses during DEG generation. The energy conversion efficiency of these devices is mainly limited by viscous dissipation and poor capillary to electrical energy conversion. Experimental data and our numerical simulations show that a small fraction of the initial kinetic energy of impinging droplets is converted into surface energy at maximum spread. The remaining energy is lost as shear work and internal kinetic energy. Even though slower impacts provide less peak power, they dissipate much less energy. For applications where the total energy is critical (as opposed to the peak power), slower impact velocities may allow extracting more energy. This may be achieved by cascading generators with small gap height between them, or even harvesting the energy of crawling droplets ${ }^{39}$. Furthermore, increasing the device charge is indeed a key element in the path of improving DEG efficiency. By reducing the impact speed and increasing the surface charge, it makes little doubt that DEG efficiency can be improved beyond the current $10 \%$.

\section{Materials and methods OpenFoam simulations}

Our simulation is performed in OpenFOAM and based on the case of breaking dam in case base of OpenFOAM $^{40}$. The simulation area is $30 \times 30 \times 40 \mathrm{~mm}^{2}$, discretized into $120 \times 160 \times 120$ elements. The fluid properties are shown in Table 2. We used a laminar flow solver and VOF explicit interface tracking.

A simulation script is available in Supplementary Information.

\section{Acknowledgements}

This work was supported by the National Natural Science Foundation of China with Grant nos. 12004078, 51950410582, 61874033 and 61674043, the Science Foundation of Shanghai Municipal Government with Grant no. 18ZR1402600, the State Key Lab of ASIC and System, Fudan University with Grant nos. 2018MS003 and 2020KF006. We also acknowledge the financial support from the National Natural Science Foundation of China (No. 51975502), GuangdongHong Kong Technology Cooperation Funding Scheme (GHP/021/19SZ), and Innovation \& Technology Fund (9440248) to Z.W.

\section{Author contributions}

A.R. proposed the research, W.X. and Z.W. measured the droplet contact line dynamics and electric current flow in DEG during droplet impact, C.W.

simulated the droplet impact, A.R. and C.W. derived the theory, all the authors discussed the results and wrote the paper conjointly.

\section{Competing interests}

The authors declare no competing interests.

Supplementary information The online version contains supplementary material available at https://doi.org/10.1038/s41378-021-00269-8.

Received: 22 January 2021 Revised: 8 April 2021 Accepted: 9 April 2021 Published online: 21 June 2021

\section{References}

1. $\mathrm{Xu}, \mathrm{W}$. et al. A droplet-based electricity generator with high instantaneous power density. Nature 578, 392-396 (2020).

2. Wu, H., Mendel, N., van den Ende, D., Zhou, G. \& Mugele, F. Energy harvesting from drops impacting onto charged surfaces. Phys. Rev. Lett. 125, 78301 (2020).

3. Xu, W. \& Wang, Z. Fusion of slippery interfaces and transistor-inspired architecture for water kinetic energy harvesting. Joule 4, 2527-2531 (2020).

4. $\mathrm{Wu}, \mathrm{H}$. et al. Charge trapping-based electricity generator (cteg): an ultrarobust and high efficiency nanogenerator for energy harvesting from water droplets. Adv. Mater. 32, 2001699 (2020).

5. Lin, Z.-H., Cheng, G., Lee, S., Pradel, K. C. \& Wang, Z. L. Harvesting water drop energy by a sequential contact-electrification and electrostatic-induction process. Adv. Mater. 26, 4690-4696 (2014).

6. Yatsuzuka, K., Mizuno, Y. \& Asano, K. Electrification phenomena of pure water droplets dripping and sliding on a polymer surface. J. Electrost. 32, 157-171 (1994).

7. Yatsuzuka, K., Higashiyama, Y. \& Asano, K. Electrification of polymer surface caused by sliding ultrapure water. In Proceedings of 1994 IEEE Industry Applications Society Annual Meeting, vol. 2, 1400-1405 (1994).

8. Zimmermann, R., Freudenberg, U., Schweiß, R., Küttner, D. \& Werner, C. Hydroxide and hydronium ion adsorption-a survey. Curr. Opin. Colloid Interface Sci. 15, 196-202 (2010).

9. Banpurkar, A. G. et al. Spontaneous electrification of fluoropolymer-water interfaces probed by electrowetting. Faraday Discuss. 199, 29-47 (2017).

10. Stetten, A. Z., Golovko, D. S., Weber, S. A. L. \& Butt, H.-J. Slide electrification: charging of surfaces by moving water drops. Soft. Matter. 15, 8667-8679 (2019).

11. Lin, Z.-H., Cheng, G., Lin, L., Lee, S. \& Wang, Z. L. Water-solid surface contact electrification and its use for harvesting liquid-wave energy. Angew. Chem. Int. Ed. 52, 12545-12549 (2013).

12. Nie, J. et al. Probing contact-electrification-induced electron and ion transfers at a liquid-solid interface. Adv. Mater. 32, e1905696 (2019).

13. $\mathrm{Wu}, \mathrm{H}$. et al. Electrically controlled localized charge trapping at amorphous fluoropolymer-electrolyte interfaces. Small 16, 1905726 (2020).

14. Wang, $X$. et al. Dynamics for droplet-based electricity generators. Nano Energy 80, 105558 (2020).

15. Wildeman, S., Visser, C. W., Sun, C. \& Lohse, D. On the spreading of impacting drops. J. Fluid Mech. 805, 636-655 (2016).

16. Chandra, S. \& Avedisian, C. On the collision of a droplet with a solid surface. Proc. R. Soc. Lond. Ser. A 432, 13-41 (1991).

17. Pasandideh-Fard, M., Qiao, Y., Chandra, S. \& Mostaghimi, J. Capillary effects during droplet impact on a solid surface. Phys. Fluids 8, 650-659 (1996). 
18. Ukiwe, C. \& Kwok, D. Y. On the maximum spreading diameter of impacting droplets on well-prepared solid surfaces. Langmuir 21, 666-673 (2005).

19. Richard, D., Clanet, C. \& Quéré, D. Contact time of a bouncing drop. Nature 417, 811-811 (2002).

20. Clanet, C., Béguin, C., Richard, D. \& Quéré, D. Maximal deformation of an impacting drop. J. Fluid Mech. 517, 199 (2004).

21. Biance, A.-L., Chevy, F., Clanet, C., Lagubeau, G. \& Quéré, D. On the elasticity of an inertial liquid shock. J. Fluid Mech. 554, 47 (2006).

22. Laan, N., de Bruin, K. G., Bartolo, D., Josserand, C. \& Bonn, D. Maximum diameter of impacting liquid droplets. Phys. Rev. Appl. 2, 044018 (2014).

23. Roisman, I. V. Inertia dominated drop collisions. ii. an analytical solution of the navier-stokes equations for a spreading viscous film. Phys. Fluids 21, 052104 (2009).

24. Xu, L., Zhang, W. W. \& Nagel, S. R. Drop splashing on a dry smooth surface Phys. Rev. Lett. 94, 184505 (2005).

25. Riboux, G. \& Gordillo, J. M. Experiments of drops impacting a smooth solid surface: a model of the critical impact speed for drop splashing. Phys. Rev. Lett. 113, 24507-24507 (2014).

26. Dhiman, R. \& Chandra, S. Rupture of thin films formed during droplet impact. Proc. R. Soc. A 466, 1229-1245 (2010).

27. Kim, H.-Y. \& Chun, J.-H. The recoiling of liquid droplets upon collision with solid surfaces. Phys. fluids 13, 643-659 (2001).

28. Okumura, K., Chevy, F., Richard, D., Quéré, D. \& Clanet, C. Water spring: a model for bouncing drops. EPL 62, 237 (2003).

29. Richard, D. \& Quéré, D. Bouncing water drops. EPL 50, 769 (2000).
30. Lubarda, V. A. \& Talke, K. A. Analysis of the equilibrium droplet shape based on an ellipsoidal droplet model. Langmuir 27, 10705-10713 (2011).

31. Lamb, H. Statics, including hydrostatics and the elements of the theory of elasticity. Bull. Amer. Math. Soc 27, 475-477 (1921).

32. Taylor, G. \& Michael, D. On making holes in a sheet of fluid. J. Fluid Mech. $\mathbf{5 8}$ 625-639 (1973).

33. Sharma, A. \& Ruckenstein, E. Dewetting of solids by the formation of holes in macroscopic liquid films. J. Colloid Interface Sci. 133, 358-368 (1989).

34. Strutt, J. W. Vi. on the capillary phenomena of jets. Proc. R. Soc. Lond. 29, 71-97 (1879).

35. Zhan, F. et al. Electron transfer as a liquid droplet contacting a polymer surface. ACS Nano 14, 17565-17573 (2020).

36. Mugele, F. \& Baret, J.-C. Electrowetting: from basics to applications. J. Phys. 17 R705 (2005).

37. Krupenkin, T. \& Taylor, J. A. Reverse electrowetting as a new approach to highpower energy harvesting. Nat. Commun. 2, 1-8 (2011).

38. Liu, Y. et al. Pancake bouncing on superhydrophobic surfaces. Nat. Phys. 10, 515-519 (2014).

39. Cheng, X., Meng, B., Han, M., Chen, H. \& Zhang, H. A high-efficiency transparent electrification-based generator for harvesting droplet energy. In 2015 Transducers-2015 18th International Conference on SolidState Sensors, Actuators and Microsystems (TRANSDUCERS), 62-65 (IEEE, 2015).

40. Greenshields, C. Openfoam v6 user guide: 2.3 breaking of a dam. https://cfd. direct/openfoam/user-guide/v6-damBreak/ (2018). Accessed 2020-12-23. 